Mathematical Research Letters 1, 409-425 (1994)

\title{
SYMMETRIC ENVELOPING ALGEBRAS, AMENABILITY AND AFD PROPERTIES FOR SUBFACTORS
}

\author{
SORIN POPA
}

\section{Introduction}

The problems and results that relate directly combinatorial aspects with analytical ones appear to play a central place in the theory of subfactors of finite Jones index.

Along these lines, a rather puzzling phenomenon that needs to be clarified is the existence of hyperfinite subfactors that can be approximated by finite dimensional commuting squares, i.e., which are AFD, but not in a standard way, i.e. not by commuting squares that are locally isomorphic to algebras of higher relative commutants.

Approximation by higher relative commutants (standard AFD) turns out to be equivalent to the amenability of the inclusion $N \subset M$, a functional analytical property which requires existence of hypertraces for all (smooth) representations of $N \subset M$ and which is also equivalent to $M$ being hyperfinite and $\left\|\Gamma_{N, M}\right\|^{2}=[M: N]$ (see 2.1 in this paper and [Po1]). As amenability can also be characterized by merely requiring existence of hypertraces for the standard representation of $N \subset M$ (see 2.1), we may conclude that standard AFD can be "encoded in a hypertrace".

Also, (3.1.3 in [Po1]) shows that the AFD (not necessarily standard) property for an inclusion implies the existence of atomic representations with hypertraces for $N \subset M$.

The purpose of this paper is to introduce a complementary approach to finite dimensional approximation of subfactors, which was partly hinted in $([\mathrm{Po} 3])$. This approach is based on the notion of symmetric enveloping algebras and symmetric Markov traces (and hypertraces) associated to $N \subset M$. Thus, we consider the universal $C^{*}$-algebra $\mathfrak{U}=C_{\text {bin }}^{*}\left(M, e_{N}, M^{\text {op }}\right)$ generated by a "normal" copy of $M$, an antisymmetric copy of it $M^{\text {op }}$ that

Received February 22, 1994.

Supported in part by NSF Grant DMS-8908281. 
commutes with $M$ and a projection $e_{N}$ that implements both the conditional expectation of $M$ onto $N$ and of $M^{\mathrm{op}}$ onto $N^{\mathrm{op}}$. This algebra has a natural irreducible representation $C^{*}\left(M, e_{N}, J M J\right)$ as left-right multiplication by $M$ on $L^{2}(M)$ and $e_{N}=\operatorname{proj}_{L^{2}(N)}$. We prove that $\mathfrak{U}$ has a unique maximal ideal and a unique trace, called symmetric Markov trace. The resulting type $\mathrm{II}_{1}$ factor $M \otimes M^{\mathrm{op}}$ is called the symmetric enveloping type $\mathrm{II}_{1}$ factor associated to $N \stackrel{e_{N}}{\subset} M$. If $M$ is hyperfinite then the enveloping type $\mathrm{II}_{1}$ factors are "thin", i.e., they are generated linearly by a product of two of its hyperfinite subfactors $R_{1}, R_{2}$, as $\left(\operatorname{span}\left(R_{1} R_{2}\right)\right)^{-}$. Thin type $\mathrm{II}_{1}$ factors need not be hyperfinite.

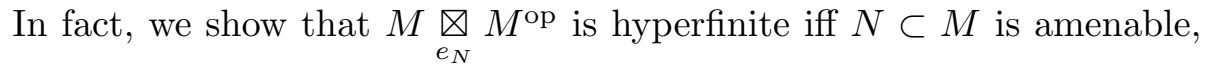
so iff $N \subset M$ is standard AFD (or, equivalently, if $\left\|\Gamma_{N, M}\right\|^{2}=[M: N]$ ). We use this to prove that the amenability for inclusions is a hereditary property i.e., if $Q \subset P$ is an extremal inclusion of type $\mathrm{II}_{1}$ factors which is embeddable in an amenable $N \subset M$ (without necessarily having $Q$ and $P$ with finite index in $N$, resp. $M$ ) then $Q \subset P$ is amenable. We then introduce the notion of symmetric Markov hypertraces for representations of $\mathfrak{U}$ on $\mathcal{B}(\mathcal{H})$, as states on $\mathcal{B}(\mathcal{H})$ that have $M, e_{N}, M^{\text {op }}$ in their centralizer, and prove that $N \subset M$ is amenable iff there exist symmetric Markov hypertraces on any representation of $\mathfrak{U}$ and also iff $\mathfrak{U}$ is simple.

Also, we show that in order for $N \subset M$ to be AFD it is sufficient to exist subfactors $Q \subset N$ with $Q^{\prime} \cap N \subset Q^{\prime} \cap M$ finite dimensional and having inclusion matrix of square norm close to $[M: N]$. If in addition $Q$ can be taken so that $[N: Q]<\infty$ then $N \subset M$ follows amenable. Finally, we show that if $N \subset M$ is AFD (not necessarily standard) then $C^{*}\left(M, e_{N}, J M J\right) \subset \mathcal{B}\left(L^{2}(M)\right)$ has symmetric Markov hypertraces and prove the later condition equivalent to a Connes-Folner type condition. We use this to construct examples of inclusions of hyperfinite type $\mathrm{II}_{1}$ factors that do not have symmetric Markov hypertraces and thus are not AFD. In fact, most of our considerations are accompanied by motivating examples.

\section{Symmetric enveloping algebras}

Let $N \subset M$ be a subfactor of finite Jones index, $[M: N]<\infty$, which we will always assume to be extremal, i.e., $[p M p: N p]=(\operatorname{tr} p)^{2}[M: N]$ for any projection $p \in N^{\prime} \cap M$, or equivalently $E_{N^{\prime} \cap M}\left(e_{0}\right) \in \mathbb{C}$, $e_{0}$ being a Jones projection for $N \subset M$. If $N \subset M \stackrel{e_{1}}{\subset} M_{1} \stackrel{e_{2}}{\subset} \ldots$ is the Jones' tower of factors associated to the inclusion then its closure $M_{\infty} \stackrel{\text { def }}{=} \underset{n \geq 1}{\cup} M_{n}$ with respect to the unique normalized trace $t r$ on $\cup_{n} M_{n}$ is a type $\bar{I}_{1}$ factor, called the enveloping algebra associated to $N \subset{ }^{n} M . M_{\infty}$ is clearly hyperfinite if and 
only if $M($ or $N)$ is hyperfinite.

In the case $N \subset M$ is strongly amenable, i.e., $M$ is hyperfinite and the standard graph of $N \subset M, \Gamma_{N, M}$, is ergodic and satisfies $\left\|\Gamma_{N, M}\right\|^{2}=[M$ : $N]$, then $N \subset M$ is antiisomorphic to $M_{1}^{\prime} \cap M_{\infty} \subset M^{\prime} \cap M_{\infty}$ (cf [Po1]). So in this case $M_{\infty}$ is generated by $M$, by a copy of $M^{\text {op }}$ that commutes with $M$ and by a projection $e_{N}$ which implements both the expectation of $M$ onto $N$ and of $M^{\mathrm{op}}$ onto $N^{\mathrm{op}}$. Equivalently, $M_{\infty}$ has an (anti)symmetry, denoted $x \mapsto x^{\mathrm{op}}$, such that $\left(x^{\mathrm{op}}\right)^{\mathrm{op}}=x,\left[M, M^{\mathrm{op}}\right]=0,\left(e_{N}\right)^{\mathrm{op}}=e_{N}$ and $M_{\infty}=M \vee\left\{e_{N}\right\} \vee M^{\mathrm{op}}$.

For an arbitrary inclusion of type $\mathrm{II}_{1}$ factors of finite index one has a similar symmetric enveloping algebra.

Definition 1.1. Let $Q \stackrel{E_{Q}}{\subset} P$ be a Markov inclusion of finite von Neumann algebras with $E_{Q}$ the trace preserving expectation (thus, there exists an orthonormal basis of $P$ over $Q,\left\{m_{j}\right\}_{j}$, such that $\Sigma m_{j} m_{j}^{*}$ is a scalar multiple of the identity (see [Po1])). Let $Q \subset P \stackrel{e_{Q}}{\subset}\left\langle P, e_{Q}\right\rangle$ be the Jones basic construction for $Q \subset P([\mathrm{~J}])$. We let $C_{\max }^{*}\left(P, e_{Q}, P^{\text {op }}\right)$ be the universal $C^{*}$-algebra containing a copy of $\left\langle P, e_{Q}\right\rangle$ and having an antiautomorphism $x \mapsto x^{\mathrm{op}}$, satisfying the conditions

(a) $\left(x^{\mathrm{op}}\right)^{\mathrm{op}}=x$

(b) $\left[P, P^{\mathrm{op}}\right]=0,\left(e_{Q}\right)^{\mathrm{op}}=e_{Q}$

(c) $P, P^{\text {op }}, e_{Q}$ generate $\mathfrak{U}$ as a $C^{*}$-algebra.

We then denote by $\mathfrak{U}=C_{\mathrm{bin}}^{*}\left(P, e_{Q}, P^{\mathrm{op}}\right)=C_{\max }^{*}\left(P, e_{Q}, P^{\text {op }}\right) / \cap \operatorname{ker} \pi$, where the intersection is over all representations $\pi$ of $C_{\max }^{*}\left(P, e_{Q}, P^{\text {op }}\right)$ for which $\pi(P), \pi\left(P^{\text {op }}\right)$ are von Neumann algebras. We call $\mathfrak{U}$ the universal symmetric enveloping $C^{*}$-algebra associated to $Q \subset P$.

Theorem 1.2. Let $N \subset M$ be an extremal inclusion of type $\mathrm{II}_{1}$ factors of finite index. Then there exists a unique positive normalized trace on $C_{\mathrm{bin}}^{*}\left(M, e_{N}, M^{\mathrm{op}}\right)$ and its associated ideal is the unique maximal ideal of $C_{\mathrm{bin}}^{*}\left(M, e_{N}, M^{\mathrm{op}}\right)$. Equivalently, any $C^{*}$-algebra $\mathfrak{U}_{0}$ which contains a copy of $\left\langle M, e_{N}\right\rangle$ and has an antisymmetry op satisfying the axioms $\left.\left.1.1 a\right), b\right)$, $c)$ and which acts on a Hilbert space so that $M, M^{\mathrm{op}}$ are weakly closed, has a unique trace.

To prove this theorem, one uses the following:

Lemma 1.3. Let $M \stackrel{e_{0}}{\supset} N \stackrel{e_{-1}}{\supset} N_{1} \supset \cdots$ be a choice of the tunnel for $N \subset M$. If $e_{1}=e_{N}, e_{2}=\left(e_{0}\right)^{\mathrm{op}}, e_{3}=\left(e_{-1}\right)^{\mathrm{op}}, \ldots$ in $C_{\max }^{*}\left(M, e_{N}, M^{\mathrm{op}}\right)$, and $M_{1}=\left\langle M, e_{1}\right\rangle, M_{2}=\left\langle M_{1}, e_{2}\right\rangle, \ldots$, then $N \subset M \subset M_{1} \subset \cdots$ is isomorphic to the Jones tower for $N \subset M$ and one has $C_{\max }^{*}\left(M, e_{N}, M^{\mathrm{op}}\right)=$ $\overline{s p}^{n} M^{\mathrm{op}}\left(\underset{k}{\cup} M_{k}\right) M^{\mathrm{op}}$. 
With this observation at hand one can check that

$$
x_{1}^{\mathrm{op}} Y x_{2}^{\mathrm{op}} \mapsto x_{1}^{\mathrm{op}} E_{M^{\prime} \cap M_{k}}(Y) x_{2}^{\mathrm{op}}, \quad x_{1,2} \in M, Y \in M_{k}
$$

defines an expectation of $\mathfrak{U}$ onto $M^{\text {op }}$ that factors through any representation of $\mathfrak{U}$. Then one gets that $\tau\left(x_{1}^{\mathrm{op}} Y x_{2}^{\mathrm{op}}\right)=\tau\left(x_{1}^{\mathrm{op}} E_{M^{\prime} \cap M_{k}}(Y) x_{2}^{\mathrm{op}}\right)$ defines a trace on any representation of $\mathfrak{U}$.

We denote by $C_{\min }^{*}\left(M, e_{N}, M^{\text {op }}\right)$ the quotient of $\mathfrak{U}$ by its trace ideal. Note that, by using 1.3 and a similar argument as in the above proof, one can show that there exits a unique conditional expectation of $\mathfrak{U}$ onto the $C^{*}$-algebra $C^{*}\left(M, M^{\mathrm{op}}\right)$ generated by $M$ and $M^{\mathrm{op}}$ in $\mathfrak{U}$. The Stinespring dilation of this expectation then shows that the subalgebra $C^{*}\left(M, M^{\mathrm{op}}\right)$ of $\mathfrak{U}$ coincides with $C_{\mathrm{bin}}^{*}\left(M, M^{\mathrm{op}}\right)$ as defined in ([EL]). In particular, if $N=M$ then $\mathfrak{U} \simeq C_{\text {bin }}^{*}\left(M, M^{\text {op }}\right)$.

By taking the GNS representation corresponding to the trace given by the above theorem we then get:

Corollary 1.4. There exists a unique (up to conjugacy) type $\mathrm{II}_{1}$ factor $U$, with a copy of $\left\langle M, e_{N}\right\rangle \subset U$ and with an antiautomorphism $x \mapsto x^{\mathrm{op}}$, such that

(a) $\left(x^{\mathrm{op}}\right)^{\mathrm{op}}=x, \forall x \in U$

(b) $\left[M, M^{\mathrm{op}}\right]=0, e_{N}^{\mathrm{op}}=e_{N}$

(c) $M, M^{\mathrm{op}}, e_{N}$ generate $U$ as a von Neumann algebra.

Moreover, if $M \stackrel{e_{0}}{\supset} N \stackrel{e_{-1}}{\supset} N_{1} \supset \cdots$ is a choice of a tunnel for $N \subset M$ and $e_{1}=e_{N}, e_{2}=\left(e_{0}\right)^{\mathrm{op}}, e_{3}=\left(e_{-1}\right)^{\mathrm{op}}, \cdots \in U$, then $N \subset M \stackrel{e_{1}}{\subset} M_{1} \subset \cdots$ is isomorphic to the Jones tower, $M_{\infty}=\overline{\cup M_{n}^{w}}$ to the usual enveloping algebra of $N \subset M$ and one has $U=\overline{s p}^{w} M_{\infty} M^{\mathrm{op}}, M_{n}^{\prime} \cap U=\left(N_{n-1}\right)^{\mathrm{op}}$, $\forall n \in \mathbb{Z}$, in particular $\left(M_{n}^{\prime} \cap U\right)^{\prime} \cap U=M_{n}, \forall n \in \mathbb{Z}$.

Definition 1.5. The type $\mathrm{II}_{1}$ factor $U$ in the above Corollary is called the symmetric enveloping type $\mathrm{II}_{1}$ factor associated to $N \subset M$. We denote it by $M \underset{e_{N}}{\otimes} M^{\mathrm{op}}$.

Thus, as the above comments show, $M \underset{e_{N}}{\underset{\mathrm{O}}{\otimes}} M^{\mathrm{op}}$ coincides with $M_{\infty}$ in the case $N \subset M$ is extremal and strongly amenable, with $N \neq M$. Note also that it coincides with $M \bar{\otimes} M^{\text {op }}$ when $N=M$.

For arbitrary (e.g., finite dimensional) Markov inclusions $Q \subset P$ one should first determine what traces on $C_{\mathrm{bin}}^{*}\left(P, e_{Q}, P^{\mathrm{op}}\right)$ are of interest for us.

Definition 1.6. A trace on the universal symmetric enveloping $C^{*}$-algebra $C_{\mathrm{bin}}^{*}\left(P, e_{Q}, P^{\mathrm{op}}\right)$ which extends the Markov trace on $\left\langle P, e_{Q}\right\rangle$ and is invariant to op is called a symmetric Markov trace. 
In some situations we can prove existence of such traces by using 1.2.

Proposition 1.7. Let $Q_{-1} \subset P_{-1}$ be a Markov inclusion. Assume there exists an extremal inclusion of type $\mathrm{II}_{1}$ factors $N \subset M$ and an embedding of $Q_{-1} \subset P_{-1}$ into $N \subset M$ as a nondegenerate commuting square. Then $C_{\mathrm{bin}}^{*}\left(P_{-1}, e_{Q_{-1}}, P_{-1}^{\mathrm{op}}\right)$ has a symmetric Markov trace. In particular, if $Q_{-1} \subset P_{-1}$ is finite dimensional and

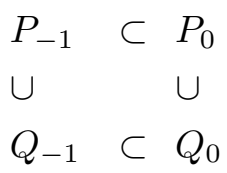

is an extremal commuting square with $\mathcal{Z}\left(P_{-1}\right) \cap \mathcal{Z}\left(P_{0}\right)=\mathbb{C}$, then $C_{\mathrm{bin}}^{*}\left(P_{-1}, e_{Q_{-1}}, P_{-1}^{\mathrm{op}}\right)$ has a symmetric Markov trace.

One should note that for general $Q \subset P$ the symmetric Markov trace on $C_{\mathrm{bin}}^{*}\left(P, e_{Q}, P^{\mathrm{op}}\right)$ (if it exists) is not necessarily unique. For instance if $Q=\mathbb{C} \subset \mathbb{C}^{n}=P$ then one has a symmetric Markov trace $\tau_{0}$ on $\mathfrak{U}=C_{\text {bin }}^{*}\left(P, e_{Q}, P^{\text {op }}\right)$ by simply taking $P, P^{\text {op }}$, act on $L^{2}(P)$ by left and respectively right multiplication, $e_{Q}$ to act as the projection onto $L^{2}(Q)$ and $\tau_{0}=\frac{1}{\operatorname{dim} P} \operatorname{Tr}_{\mathcal{B}\left(L^{2}(P)\right)}$. The image of $\mathfrak{U}$ under $\pi_{\tau_{0}}$ is then $\mathcal{B}\left(L^{2}(P)\right)=$ $M_{n \times n}(\mathbb{C})$. But if we take $Q \subset P$ to be the inclusion $Q_{-1} \subset P_{-1}$ in a commuting square like in 1.7, with $P_{0}=M_{n \times n}(\mathbb{C})$ and $Q_{0}$ an exotic Jones-de la Harpe orthogonal maximal abelian subalgebra in $M_{n \times n}(\mathbb{C})$, with $n$ a prime number, then the trace $\tau_{1}$ produced by the proof of 1.7 is different from $\tau_{0}$. In fact $\pi_{\tau_{1}}(\mathfrak{U}) \neq M_{n \times n}(\mathbb{C})$.

There is no a priori reason for an arbitrary finite dimensional inclusion $Q \subset P$ to have an associated Markov trace on $C_{\mathrm{bin}}^{*}\left(P, e_{Q}, P^{\mathrm{op}}\right)(=$ $C_{\text {max }}^{*}\left(P, e_{Q}, P^{\text {op }}\right)$ in this case $)$. It is not hard to see though that if $C_{\text {bin }}^{*}(P$, $\left.e_{Q}, P^{\mathrm{op}}\right)$ does have one then $C_{\mathrm{bin}}^{*}\left(p P p, e_{p Q p},(p P p)^{\mathrm{op}}\right)$ and $C_{\mathrm{bin}}^{*}\left(P \otimes M_{n \times n}\right.$ $\left.(\mathbb{C}), e_{Q \otimes M_{n \times n}(\mathbb{C})},\left(P \otimes M_{n \times n}(\mathbb{C})\right)^{\text {op }}\right)$ have one. So, the existence of such a trace really depends only on the bipartite inclusion graph of $Q \subset P, T_{Q \subset P}$. We can thus call such traces symmetric Markov traces associated to $T_{Q \subset P}$.

Problem 1.8. Do all bipartite graphs have symmetric Markov traces?

Note that by 1.7 the failure for some bipartite graph $T$ to have such a trace would imply that the commuting square problem has no solution for $T$. We do not however have any example of such a graph.

The representation of $C_{\mathrm{bin}}^{*}\left(P, e_{Q}, P^{\mathrm{op}}\right)$ on $L^{2}(P)$ works in fact for arbitrary inclusions.

Corollary 1.9. Let $\mathfrak{U}_{0}=C^{*}\left(P, e_{Q}, J P J\right)$ be the $C^{*}$-algebra generated in $\mathcal{B}\left(L^{2}(M)\right)$ by $P, J_{P} P J_{P}=P^{\text {op }}$ and $e_{Q}=\operatorname{proj}_{L^{2}(Q)}$ and denote $x^{\text {op }} \stackrel{\text { def }}{=}$ 
$J x^{*} J, x \in \mathfrak{U}_{0}$. Then $\mathfrak{U}_{0}$, with $\left\langle P, e_{Q}\right\rangle \subset \mathfrak{U}_{0}$ and the above op is a representation of $C_{\mathrm{bin}}^{*}\left(P, e_{Q}, P^{\mathrm{op}}\right)$. Moreover, if $\mathcal{Z}(Q) \cap \mathcal{Z}(P)=\mathbb{C}$ than this representation is irreducible. Also, if $Q \subset P$ is an inclusion of type $\mathrm{II}_{1}$ factors then there exists a unique trace on $C^{*}\left(P, e_{Q}, J P J\right)$.

In the case $M$ is the hyperfinite type $\mathrm{II}_{1}$ factor the above results justify considering the following property for type $\mathrm{II}_{1}$ factors.

Definition 1.10. A type $\mathrm{II}_{1}$ factor $U$ is called thin if there exist two copies of the hyperfinite factor $R_{1}, R_{2} \subset U$ such that $U=\overline{\mathrm{sp}}^{w} R_{1} R_{2}$.

Thus, to each extremal inclusion of hyperfinite type $\mathrm{II}_{1}$ factors $N \subset M$ one associates a thin type $\mathrm{II}_{1}$ factor whose isomorphism class only depends on the isomorphism class of $N \subset M$. Note that we have a natural group morphism from $\operatorname{Aut}(N \subset M)$ into the automorphism of $M \underset{e_{N}}{\otimes} M$ that commute with op, leave $e_{N}$ fixed and $M, N$ invariant, by associating to $\sigma$ in $\operatorname{Aut}(N \subset M)$ the automorphism of $M \underset{e_{N}}{\otimes} M$ that sends $x_{1} y_{1}^{\mathrm{op}} e_{N} x_{2} y_{2}^{\mathrm{op}} e_{N} \cdots$ into $\sigma\left(x_{1}\right) \sigma\left(y_{1}\right)^{\mathrm{op}} e_{N} \sigma\left(x_{2}\right) \sigma\left(y_{2}\right)^{\mathrm{op}} e_{N} \ldots$. This morphism implements an injective morphism from $\operatorname{Aut}(N \subset M) / \operatorname{Int}(\mathcal{N}(N))$ into $\operatorname{Out}\left(M \underset{e_{N}}{\otimes} M^{\mathrm{op}}\right)$, where $\mathcal{N}(N)$ is the normalizer of $N$ in $M$.

\section{Amenability and standard AFD}

A first motivation for studying the symmetric enveloping algebras and their Markov traces comes from the following:

Theorem 2.1. Let $N \subset M$ be an extremal inclusion of type $\mathrm{II}_{1}$ factors of finite index. The following conditions are equivalent:

(i) $N \subset M$ is amenable ([Po1]).

(ii) $N \subset M$ has the standard AFD property, i.e., it can be approximated by finite dimensional commuting squares which are locally isomorphic to reduced algebras of higher relative commutants (in the sense of 4.4 .1 in $[\mathrm{Po} 1])$.

(iii) $\forall x_{1}, \ldots, x_{n} \in M, \forall \varepsilon>0, \exists$ a subfactor $P \subset N$ of finite index such that $x_{i} \in P^{\prime} \cap M$.

(iv) $M \underset{e_{N}}{\bigotimes} M^{\mathrm{op}}$ is hyperfinite.

(v) The standard representation $\mathcal{N}^{\text {st }} \subset \mathcal{M}^{\text {st }}$ of $N \subset M$ has a $(N \subset M)$ hypertrace.

(vi) $M$ is hyperfinite and $\left\|\Gamma_{N, M}\right\|^{2}=[M: N]$.

(vii) $M$ is hyperfinite and $\Gamma_{N, M}$ satisfies the Folner condition ([Po2]). 
Note that in the case $N=M$ the equivalence (i) $\Leftrightarrow$ (ii) simply states that $M$ is amenable (or injective) iff $M \otimes M^{\text {op }}$ is hyperfinite, which is an equivalent form of Connes' fundamental theorem.

(i) $\Rightarrow$ (ii) is proved in [Po1]. Then (ii) $\Rightarrow$ (iii) is straightforward, by (4.4.1 in [Po1]) and by defining the subfactor $P$ as $\left\{\underset{j \in I_{0}}{\oplus} \theta_{i_{0} j}(x) \mid x \in\right.$ $\left.N_{k_{i_{0}}}^{i_{0}} s_{i_{0}}\right\}$, where $\theta_{i j}: N_{k_{i}}^{i} s_{i} \rightarrow N_{k_{j}}^{j} s_{j}$ are isomorphisms and the rest of the notations are from (4.4.1 in [Po1]). To prove (iii) $\Rightarrow$ (iv) one uses that if $P \subset N \subset M$ is so that $[M: P]<\infty$ then $P^{\prime} \cap\left(P^{\mathrm{op}}\right)^{\prime}$ is finite dimensional in $M \underset{e_{N}}{\otimes} M^{\mathrm{op}}$ (since $\left.\left[P^{\mathrm{op}} \cap M \underset{e_{N}}{\otimes} M^{\mathrm{op}}: P\right]<\infty\right)$. Then (iv) $\Rightarrow$ (v) as follows: let $U=M \underset{e_{N}}{\otimes} M^{\text {op }}$ be represented by left multiplication on $L^{2}(U)$ and define $\mathcal{M}^{\text {st }}=M \vee J_{U} M J_{U}, \mathcal{N}^{\text {st }}=N \vee J_{U} M J_{U}$. It is easy to see that the embedding of $N \subset M$ into $\mathcal{N}^{\text {st }} \subset \mathcal{M}^{\text {st }}$ is isomorphic to the standard representation of $N \subset M$ as defined in [Po1], taken with infinite multiplicity. Thus, if $\Phi: \mathcal{B}\left(L^{2}(U)\right) \rightarrow U$ is a conditional expectation onto $U$ then $\Phi\left(\mathcal{M}^{\text {st }}\right) \subset U$ commutes with $M^{\text {op }}$, so $\Phi\left(\mathcal{M}^{\text {st }}\right)=\left(M^{\text {op }}\right)^{\prime} \cap U=M$, and $\Phi\left(\mathcal{N}^{\text {st }}\right)$ commutes with $\left\langle M^{\text {op }}, e_{N}\right\rangle$, so $\Phi\left(\mathcal{N}^{\text {st }}\right)=\left\langle M, e_{N}\right\rangle^{\prime} \cap U=N$. Thus $\tau \circ \Phi$ is a $(N \subset M)$-hypertrace for $\mathcal{N}^{\text {st }} \subset \mathcal{M}^{\text {st }}$.

Further on, (v) $\Rightarrow$ (vi) (by 2.4 and 4.4 .1 in [Po1]) and (vii) $\Rightarrow$ (i) is proved in [Po2]. Finally, (vi) $\Rightarrow$ (vii) by using that $N_{k}^{\prime} \cap N \subset N_{k}^{\prime} \cap M$ has inclusion graph $T_{k}$ with $\left\|T_{k}\right\|^{2} \geq[M: N]-\varepsilon=\tau\left(e_{0}\right)^{-1}-\varepsilon$, where $e_{0} x e_{0}=E_{N_{k}^{\prime} \cap N_{1}}(x) e_{0}$ for $x \in N_{k}^{\prime} \cap N$, and that $\operatorname{sp}\left(N_{k}^{\prime} \cap N\right) e_{0}\left(N_{k}^{\prime} \cap N\right)$ is "almost equal" to $N_{k}^{\prime} \cap M$.

The above condition (iv) enables us to prove the hereditarity properties for the amenability of inclusions that were missing until now from this theory.

Corollary 2.2. Let $M$ be a hyperfinite type $\mathrm{II}_{1}$ factor and $N \subset M$ an amenable subfactor (i.e. with $\left\|\Gamma_{N, M}\right\|^{2}=[M: N]$ ).

(a) If $Q \subset P$ is an inclusion of type $\mathrm{II}_{1}$ factors embedded in $N \subset M$ as a commuting square such that $\operatorname{sp} P N$ is an algebra (equivalently, $\operatorname{sp} P N=\operatorname{sp} N P)$ then $\left\|\Gamma_{Q, P}\right\|^{2}=[P: Q]$. In particular, if $Q \subset P$ is embedded in $N \subset M$ as a commuting square and $[P: Q]=[M: N]$ then $\left\|\Gamma_{Q, P}\right\|^{2}=[P: Q]=[M: N]=\left\|\Gamma_{N, M}\right\|^{2}$.

(b) If $N \subset M \subset M_{1} \cdots$ is the Jones' tower and $p \in M_{i}^{\prime} \cap M_{j}$ then $\left\|\Gamma_{M_{i} p, p M_{j} p}\right\|^{2}=\left[p M_{j} p: M_{i} p\right]$.

Note that in the previous Corollary, we do not require $[M: P]$ to be finite! By taking $Q=N$ in the above we get:

Corollary 2.3. If $N \subset P \subset M$ are inclusions of hyperfinite type $\mathrm{II}_{1}$ factors and $\left\|\Gamma_{N, M}\right\|^{2}=[M: N]$ then $\left\|\Gamma_{N, P}\right\|^{2}=[P: N]$ and $\left\|\Gamma_{P, M}\right\|^{2}=$ 
$[M: P]$.

In the case $N \subset M$ has finite depth (respectively has subexponential growth [Po1]), the similar implication on the finite depth (resp. subexponential growth) of the intermediate subfactors, as well as the analogue of 2.2(b), are proved in [Bi2] (resp. [Hi]), for arbitrary (not necessarily hyperfinite) subfactors.

The proof of 2.2 follows from Theorem 2.1, Connes' hereditarity for hyperfiniteness and the following:

Lemma 2.4. Assume $Q \subset P$ is an inclusion of type $\mathrm{II}_{1}$ factors embedded in $N \subset M$ as a commuting square.

(a) If $\operatorname{sp} P N=\operatorname{sp} N P$ then $P \underset{e_{Q}}{\otimes} P^{\text {op }}$ is naturally embedded as the weakly closed *-subalgebra in $M \underset{e_{N}}{\square} M^{\mathrm{op}}$ generated by $P, P^{\mathrm{op}}$ and $e_{N}$. Moreover, this embedding is unital iff $[M: N]=[P: Q]$, in which case $\operatorname{sp} P N=\operatorname{sp} N P$ is redundant.

(b) If $p \in N^{\prime} \cap M$ and $(N p \subset p M p) \stackrel{\text { notation }}{=}(S \subset R)$ then $R \underset{e_{S}}{\otimes} R^{\text {op }}$ is naturally embedded as the weakly closed *-subalgebra of $M \underset{e_{N}}{\otimes} M^{\mathrm{op}}$ generated by $p p^{\mathrm{op}}\left(M \otimes M^{\mathrm{op}}\right) p p^{\mathrm{op}}$ and by $(\operatorname{trp})^{-1} p p^{\mathrm{op}} e_{N} p p^{\mathrm{op}}$.

(c) If $[M: Q]<\infty$ then $M \otimes M^{\mathrm{op}}$ is naturally embedded as a unital von Neumann subalgebra of $M \underset{e_{Q}}{\otimes} M^{\mathrm{op}}$ by taking $e_{N}=\lambda \Sigma m_{j} e_{Q} m_{j}^{*}=$ $\lambda \Sigma m_{j}^{\mathrm{op}} e_{Q} m_{j}^{\mathrm{op} *}$, where $\left\{m_{j}\right\}_{j}$ is an orthonormal basis of $N$ over $Q$ and $\lambda=[N: Q]^{-1}$. In particular, $\left(Q^{\prime} \cap M\right) \vee\left\{e_{N}\right\} \vee\left(Q^{\prime} \cap M\right)^{\mathrm{op}} \subset$ $Q^{\prime} \cap\left\langle M, e_{Q}\right\rangle=Q^{\prime} \cap Q^{\mathrm{op}^{\prime}}$.

The following shows that the finite depth condition has no chance of satisfying similar hereditarity properties.

Example 2.5. Let $P_{0}$ be a copy of the hyperfinite type $\mathrm{II}_{1}$ factor and $G$ a finitely generated amenable group with nonergodic Cayley graph with respect to some set of generators $g_{0}=\mathrm{id}, g_{1}, \ldots, g_{n}$ (cf. KaimanovitchVershik, [Po1]). Let $\sigma_{0}$ be a properly outer action of $G$ on $P_{0}$ and $P=$ $P_{0} \rtimes_{\sigma_{0}} G$. Let $\sigma$ be the inner action $\operatorname{Ad} u_{g}, g \in G$, of $G$ on $P$. Let $\left(N^{\sigma} \subset\right.$ $\left.M^{\sigma}\right) \simeq\left(P \subset P \otimes M_{(n+1) \times(u+1)}(\mathbb{C})\right)$ be the diagonal locally trivial subfactor associated to $\sigma, P$ and $g_{0}, g_{1}, \ldots, g_{n}$, like in [Po1]. Let $N^{\sigma_{0}} \subset M^{\sigma_{0}}$ be the subfactor associated in a similar way to $\sigma_{0}, P_{0}$ and $g_{0}, \ldots, g_{n}$. Note that one has an obvious commuting square

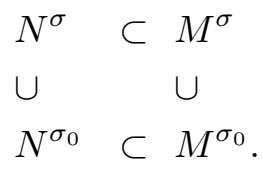


Yet $N^{\sigma} \subset M^{\sigma}$ has finite depth while $N^{\sigma_{0}} \subset M^{\sigma_{0}}$ has infinite depth and nonergodic graph. In particular its graph is not strongly amenable.

Note that if $[M: P]<\infty$ in 2.2 then indeed if $N \subset M$ has finite depth then $Q \subset P$ has finite depth as well, regardless of $M$ being hyperfinite or not (see 2.7 below). However, we cannot weaken the condition on the hyperfiniteness of $M$ in 2.2 if we allow $[M: P]=\infty$.

Example 2.6. If in 2.5 we take $G$ to be nonamenable and otherwise proceed with the same construction as in 2.5, then $N^{\sigma} \subset M^{\sigma}$ has finite depth and $N^{\sigma_{0}} \subset M^{\sigma_{0}}$ has nonamenable graph. But this time $M^{\sigma}$ is nonhyperfinite. Moreover, since $\Gamma_{N^{\sigma_{0}}, M^{\sigma_{0}}}$ is nonamenable, $M^{\sigma_{0}} \underset{e_{N}}{\square} M^{\sigma_{0}}$ is a nonhyperfinite, yet thin, type $\mathrm{II}_{1}$ factor (cf. 2.1).

We can however prove some equivalent characterizations of the amenability of an inclusion which have "finite depth" versions as well. To state them recall from ([Po1]) that a representation $\mathcal{N} \subset \mathcal{M}$ of $N \subset M$ is exact if $\mathcal{M}=M \vee P$, where $P=M^{\prime} \cap \mathcal{N}$. If $\mathcal{M}$ is also atomic then each of its direct summands $\mathcal{B}(\mathcal{H})$ is an irreducible $M-P$ correspondence (bimodule). Also, in $([\mathrm{Po} 1])$ we denoted by $\oplus \mathcal{B}(\mathcal{K})=\mathcal{N}^{u f} \subset \mathcal{M}^{u f}=\oplus \mathcal{B}(\mathcal{H})$ the direct sum of all exact atomic representations for which $\operatorname{dim}_{M} \mathcal{H} \operatorname{dim}_{P} \mathcal{H}$ is finite and by $A^{u}=A_{N, M}^{u f}$ its inclusion matrix. Note that $\mathcal{N}^{s t} \subset \mathcal{M}^{s t}$ is a direct summand of this representation, so that $\Gamma_{N, M}$ is a direct summand (or connected component) of $A^{u}$ and $\left\|\Gamma_{N, M}\right\| \leq\left\|A^{u}\right\|$. We've seen in 2.1 that if $M$ is hyperfinite then the amenability of $N \subset M$ is equivalent to $\left\|\Gamma_{N, M}\right\|^{2}=[M: N]$. We will show that $\left\|A^{u}\right\|^{2}=[M: N]$ is in fact sufficient for $N \subset M$ to be amenable, which in turn implies that any direct summand of $A^{u}$ has square norm equal to $[M: N]$. Also, we will consider atomic representations for which only the weaker condition $\operatorname{dim}_{P} \mathcal{H}<\infty$ is satisfied.

Proposition 2.7. Let $N \subset M$ be an extremal inclusion of hyperfinite type $\mathrm{II}_{1}$ factors. The following conditions are equivalent:

(i) $N \subset M$ is amenable.

(ii) $N \subset M$ has an exact atomic representation $\mathcal{N} \subset \mathcal{M}$, with $\operatorname{dim}_{P} \mathcal{H}<$ $\infty$ for all direct summands $\mathcal{B}(\mathcal{H})$ of $\mathcal{M}$, such that $\left\|T_{\mathcal{N} \subset \mathcal{M}}\right\|^{2}=[M:$ $N]$.

(iii) There exists a matricial inclusion (see [PiPo2] for the definition) of hyperfinite type $\mathrm{II}_{1}$ von Neumann algebras $Q \stackrel{E}{\subset} P$, with discrete centers, trace preserving expectation $E$ and $\left\|T_{Q \subset P}\right\|^{2}=[M: N]$, and a nondegenerate commuting square embedding of $N \subset M$ into $Q \subset P$. 
Proposition 2.8. Let $N \subset M$ be an extremal inclusion of arbitrary type $\mathrm{II}_{1}$ factors. The following conditions are equivalent:

(i) $\Gamma_{N, M}$ is finite (respectively is amenable, respectively is strongly amenable).

(ii) $\mathcal{N}^{\text {uf }} \subset \mathcal{M}^{\text {uf }}$ has an irreducible direct summand $\mathcal{N} \subset \mathcal{M}$ with finite inclusion matrix (respectively of square norm equal to the index, respectively ergodic and of square norm equal to the index).

(iii) All irreducible direct summands of $\mathcal{N}^{u f} \subset \mathcal{M}^{u f}$ have finite inclusion matrix (respectively of square norm equal to the index, respectively ergodic and of square norm equal to the index).

Note that condition (iii) in 2.7 doesn't require $M$ to have finite index in the direct summands of $P$. Also, the trace on $P$ in (iii) may be infinite, but it will be automatically finite if $P$ has finite center. Note that since $Q \subset P$ is matricial and has an orthonormal basis $\left\{m_{j}\right\}_{j}$ satisfying $\sum_{j} m_{j} m_{j}^{*}=\operatorname{Ind} E=$ $[M: N]$ (since it contains $N \subset M$ ), it follows like in $[\mathrm{J}]$ that the trace on $P$ is given by weights $\left(a_{k}\right)_{k}$ on the minimal central projections of $P$, satisfying $T T^{t}\left(a_{k}^{1 / 2}\right)_{k}=[M: N]\left(a_{k}^{1 / 2}\right)_{k}$ (see also [PiPo2]). The trace is infinite when $\sum_{k} a_{k}=\infty$. The proof of (ii) and (iii) in 2.7 is easier to explain in the case $T_{\mathcal{N} \subset \mathcal{M}}$ respectively $T_{Q \subset P}$ are finite. By taking the embedding of $N \subset M$ into $\left(M^{\prime} \cap \mathcal{N}\right)^{\prime} \cap \mathcal{N}=Q \subset P=\left(M^{\prime} \cap \mathcal{N}\right)^{\prime} \cap \mathcal{M}$ we see that to prove (ii) it is sufficient to prove (iii). But if $\operatorname{dim} \mathcal{Z}(P)<\infty$ then we see that $(Q \subset P)^{\text {op }} \simeq\left(\left\langle P, e_{Q}^{P}\right\rangle^{\prime} \cap P_{\infty} \subset P^{\prime} \cap P_{\infty}\right)$ so that if $N \subset M$ is embedded in $Q \subset P$ then $M \underset{e_{N}}{\otimes} M^{\mathrm{op}}$ is embedded in $P_{\infty}$ which is hyperfinite, thus $M \underset{e_{N}}{\bigotimes} M^{\mathrm{op}}$ is hyperfinite and 2.1 applies.

The proof of 2.8 can be best understood by first noting a simple fact which relates the growth properties of the inclusions in a commuting square of finite index. In this statement, by "standard graph" of a Markov inclusion of type $\mathrm{II}_{1}$ von Neumann algebras with finite dimensional centers we simply mean the bipartite graph that describes the inclusions of the higher relative commutants in the Jones tower.

Lemma 2.9. Let $S \subset R$ be embedded in $Q \subset P$ as a nondegenerate commuting square of Markov inclusions of finite ([PiPo1]) index, where $P, Q, R, S$ are type $\mathrm{II}_{1}$ von Neumann algebras with finite dimensional centers. If $R_{n}$ and $P_{n}$ denote the algebras in the Jones tower for the two inclusions then we have: (a) sup $\operatorname{dim} \mathcal{Z}\left(S^{\prime} \cap R_{n}\right)<\infty$ iff sup $\operatorname{dim\mathcal {Z}}\left(Q^{\prime} \cap P_{n}\right)<\infty$. (b) $\lim \operatorname{dim}\left(S^{\prime} \cap R_{n}\right)^{1 / n}=\lim \operatorname{dim}\left(Q^{\prime} \cap P_{n}\right)^{1 / n}$. In particular, the standard graphs of $S \subset R$ and $Q \subset P$ have the same norm. (c) $\operatorname{dim} \mathcal{Z}\left(S^{\prime} \cap R_{\infty}\right)$ is finite iff $\operatorname{dim} \mathcal{Z}\left(Q^{\prime} \cap P_{\infty}\right)$ is finite. 
To prove 2.8 consider first a representation of $N \subset M$ with finite dimensional center as in (ii). Then $\left(M^{\prime} \cap \mathcal{N}\right)^{\prime} \cap \mathcal{N}=Q \subset P=\left(M^{\prime} \cap \mathcal{N}\right)^{\prime} \cap \mathcal{M}$ is a matricial inclusion of type $\mathrm{II}_{1}$ von Neumann algebras with finite inclusion matrix and $N \subset M$ sits into it with finite index, in particular $\sup \operatorname{dim} \mathcal{Z}\left(Q^{\prime} \cap P_{n}\right)<\infty$ and so Lemma 2.9 applies. The other implications are similar.

Finally, to prove 2.9 note that we have $\sup \operatorname{dim} \mathcal{Z}\left(S^{\prime} \cap P_{n}\right)<\infty$, because $\operatorname{Ind} E_{Q^{\prime} \cap P_{n}}^{S^{\prime} \cap P_{n}} \leq \operatorname{Ind} E_{Q^{\prime}}^{S^{\prime}}=\operatorname{Ind} E_{S}^{Q}<\infty$. Since $\operatorname{Ind} E_{S^{\prime} \cap R_{n}}^{S^{\prime} \cap P_{n}} \leq \operatorname{Ind} E_{P_{n}}^{R_{n}}=$ $\operatorname{Ind} E_{P}^{R}<\infty$ we deduce that $\sup \operatorname{dim} \mathcal{Z}\left(S^{\prime} \cap R_{n}\right)<\infty$ which proves (a). Then (b) and (c) are easy to prove by the same type of arguments and by using (1.3.5 in [Po1]).

\section{Symmetric hypertraces and nonstandard AFD}

We will now discuss the property for an inclusion of type $\mathrm{II}_{1}$ factors $N \subset M$ of being approximable by finite dimensional commuting squares not coming from higher relative commutants. Some of the considerations that we will present appear already in $([\mathrm{Po} 3])$, but with a slightly different terminology and without giving the proper motivation. To give such motivation let us consider the following:

Example 3.1. Let

$$
\begin{array}{lll}
P_{-1} & \subset & P_{0} \\
\cup & & \cup \\
Q_{-1} & \subset & Q_{0}
\end{array}
$$

be an extremal commuting square of finite dimensional Markov inclusions, with $\mathcal{Z}\left(P_{-1}\right) \cap \mathcal{Z}\left(P_{0}\right)=\mathbb{C}, \mathcal{Z}\left(Q_{-1}\right) \cap \mathcal{Z}\left(Q_{0}\right)=\mathbb{C}$. Let

$$
\begin{aligned}
& P_{-1} \subset P_{0} \stackrel{e_{1}}{\subset} P_{1} \subset \cdots \\
& \cup \quad \cup \quad \cup \\
& Q_{-1} \subset Q_{0} \stackrel{e_{1}}{\subset} Q_{1} \subset \cdots
\end{aligned}
$$

be its associated tower of commuting squares, obtained by iterating the basic construction. Let $Q_{\infty}=\overline{\cup_{n} Q_{n}} \subset \overline{\cup_{n} P_{n}}=P_{\infty}$, which is an extremal inclusion of type $\mathrm{II}_{1}$ factors with $\left[P_{\infty}: Q_{\infty}\right]=\left\|T_{Q_{-1} \subset P_{-1}}\right\|^{2}([\mathrm{We}])$. By $[\mathrm{H}]$ there exist commuting squares $(*)$ for which $\Gamma_{Q_{\infty}, P_{\infty}}=A_{\infty}$, in particular $Q_{\infty}^{\prime} \cap P_{\infty}=\mathbb{C}$, while $2+\sqrt{5}>\left\|T_{Q_{-1} \subset P_{-1}}\right\|^{2}=\left[P_{\infty}: Q_{\infty}\right]>\left\|A_{\infty}\right\|^{2}=4$. 
By 2.1, it thus follows that the associated symmetric enveloping type $\mathrm{II}_{1}$ factor is nonhyperfinite (yet thin).

More properties related to this example are given by the next:

Proposition 3.2. If $\left(Q_{i} \subset P_{i}\right)_{i \geq-1}$ and $\left(Q_{\infty} \subset P_{\infty}\right) \stackrel{\text { notation }}{=}(N \subset M)$ are as in 3.1 then we have:

$1^{\circ} \cdot \operatorname{Alg}\left(P_{n}, e_{N}, J_{M} P_{n} J_{M}\right) \ni$

$x \mapsto \pi_{m}^{n}(x)=e_{P_{m}} x e_{P_{m}} \in e_{P_{m}} \mathcal{B}\left(L^{2}(M)\right) e_{P_{m}} \simeq \mathcal{B}\left(L^{2}\left(P_{m}\right)\right)$

is a finite dimensional representation of

$$
\operatorname{Alg}\left(P_{n}, e_{N}, J_{M} P_{n} J_{M}\right) \subset \mathcal{B}\left(L^{2}(M)\right), \text { with }
$$

$\lim _{m \rightarrow \infty} \varphi_{m}(T)=\tau(\pi(x))$, where $\pi: C^{*}\left(M, e_{N}, J M J\right) \rightarrow M \underset{e_{N}}{\bigotimes} M^{\mathrm{op}}$, $\varphi_{m}(T)=\frac{1}{\operatorname{dim} P_{m}} \operatorname{Tr}_{\mathcal{B}\left(L^{2}\left(P_{m}\right)\right)}\left(e_{P_{m}} T e_{P_{m}}\right)$ and $\tau$ is the trace on $M \underset{e_{N}}{\bigotimes}$ $M^{\mathrm{op}}$. Thus, the algebras $\operatorname{Alg}\left(P_{n}, e_{N}, J P_{n} J\right)$ are diagonalizable.

$2^{\circ}$. The state $\varphi(T)=\lim _{n \rightarrow \omega} \varphi_{n}(T)$ on $\mathcal{B}\left(L^{2}(M)\right)$ satisfies $\varphi(T x)=$ $\varphi(x T), \forall T \in \mathcal{B}\left(L^{2}(M)\right), \forall x \in C^{*}\left(M, e_{N}, J M J\right)$.

$3^{\circ}$. If $\mathcal{M}_{n}=J P_{n} J^{\prime} \cap \mathcal{B}\left(L^{2}(M)\right), \mathcal{N}_{n}=J\left\langle P_{n}, e_{N}\right\rangle J^{\prime} \cap \mathcal{B}\left(L^{2}(M)\right)$ then $N \subset M$ is represented in $\mathcal{N}_{n} \subset \mathcal{M}_{n}$ and there exists a $(N \subset M)$ hypertrace on $\oplus \mathcal{N}_{n} \subset \oplus \mathcal{M}_{n}$.

$4^{\circ} . M \otimes M^{\mathrm{op}}$ can be embedded in the Feldman-ultrapower type $\mathrm{II}_{1}$ factor $\prod_{n \rightarrow \omega}^{e_{N}} \mathcal{B}\left(L^{2}\left(P_{n}\right)\right)$.

5.. If $\Gamma_{N, M}$ is finite then for any $n$ there exists a subfactor of finite index $S$ in $N$ such that $S^{\prime} \cap M$ is finite dimensional and $P_{n} \subset$ $S^{\prime} \cap M$. In particular, $\operatorname{Alg}\left(P_{n}, e_{N}, J_{M} P_{n} J_{M}\right) \subset \mathcal{B}\left(L^{2}(M)\right)$ is finite dimensional for each $n$.

$6^{\circ}$. If $\left\|\Gamma_{N, M}\right\|^{2}<[M: N]$ (i.e. $\Gamma_{N, M}$ is not amenable) then we also have:

(a) $\pi\left(\operatorname{Alg}\left(P_{n}, e_{N}, J_{M} P_{n} J_{M}\right)\right)=\operatorname{Alg}\left(P_{n}, e_{N}, P_{n}^{\text {op }}\right)$ is a nonnuclear, subalgebra of $M \underset{e_{N}}{\bigotimes} M^{\mathrm{op}}$ and $\operatorname{Alg}\left(P_{n}, e_{N}, J_{M} P_{n} J_{M}\right)$ is a nonnuclear, diagonalizable subalgebra in $\mathcal{B}\left(L^{2}(M)\right)$. In particular, they are both infinite dimensional.

(b) $M \underset{e_{N}}{\otimes} M^{\mathrm{op}}$ is a thin but nonhyperfinite type $\mathrm{II}_{1}$ factor.

To prove $2^{\circ}$ note that $\varphi(x T)=\varphi(T x), \forall x \in \cup P_{n}, x=e_{N}, x \in$ $\cup J_{M} P_{n} J_{M}$ and $\forall T \in \mathcal{B}\left(L^{2}(M)\right)$. Since $\varphi_{\mid M}=\tau$, by [C2] we will have the equality for all $x \in M \cup J M J$. Then $1^{\circ}$ follows from $2^{\circ}, 3^{\circ}$ is just 3.1 .3 
of $[\mathrm{Po} 1]$ and $4^{\circ}$ is a consequence of $1^{\circ}$. Next, $5^{\circ}$ follows from the bicommutant relation of $M$ in $M_{\infty}$ for finite depth subfactors and from the fact that the enveloping algebra $S_{\infty}^{c}$ of $Q_{n} \subset P_{n}$ is a subalgebra of $M_{\infty}$ that contains $N^{\prime} \cap M_{\infty}$ as a subfactor of finite [PiPo1] index. Thus, if we let $S=S_{\infty}^{c^{\prime}} \cap M_{\infty}$ then $S$ satisfies the condition. Finally, $6^{\circ}$ is a consequence of 2.1 and [C1].

Related to property $3.2 .5^{\circ}$ above, note that for the standard commuting squares of higher relative commutants, as also emphasized in $2.4(\mathrm{c})$, we have:

Proposition 3.3. Let $N \subset M$ be an extremal inclusion of type $\mathrm{II}_{1}$ factors and $M \stackrel{e_{0}}{\supset} N \stackrel{e_{-1}}{\supset} N_{1} \supset \cdots \supset N_{k}$ a choice of the tunnel up to some $k$. Then $\operatorname{Alg}\left(N_{k}^{\prime} \cap M, e_{N}, J_{M}\left(N_{k}^{\prime} \cap M\right) J_{M}\right) \subseteq N_{k}^{\prime} \cap J_{M} N_{k}^{\prime} J_{M}=$ $N_{k}^{\prime} \cap\left\langle M, e_{N}, J e_{0} J, \ldots, J e_{-k+1} J\right\rangle$ in $\mathcal{B}\left(L^{2}(M)\right)$. In particular, $\operatorname{Alg}\left(N_{k}^{\prime} \cap\right.$ $\left.M, e_{N},\left(N_{k}^{\prime} \cap M\right)^{\mathrm{op}}\right) \subseteq N_{k}^{\prime} \cap\left(N_{k}^{\mathrm{op}}\right)^{\prime}=N_{k}^{\prime} \cap M_{k+1}$ in $M \underset{e_{N}}{\bigotimes} M^{\mathrm{op}}$, where $M_{k+1}=\left\langle M, e_{N}, e_{0}^{\mathrm{op}}, \ldots, e_{-k+1}^{\mathrm{op}}\right\rangle$. We have everywhere equalities when $N \subset M$ has finite depth and $k$ is larger than the depth.

Remark 3.4. If $Q \subset P$ is a finite dimensional Markov inclusion with standard inclusion graph $T_{Q \subset P}$, i.e., with $T_{Q \subset P}$ equal to the graph of a subfactor, then $T_{Q \subset P}$ has a finite dimensional symmetric Markov trace (i.e., a symmetric Markov trace $\tau$ on $\mathfrak{U}=C_{\text {bin }}^{*}\left(P, e_{Q}, P^{\text {op }}\right)$ with $\pi_{\tau}(\mathfrak{U})$ finite dimensional). Also, if

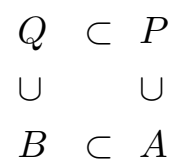

is a commuting square with $B \subset A$ a Markov inclusion of same index as $Q \subset P$, then $T_{B \subset A}$ has a finite dimensional symmetric Markov trace as well. This fact and 3.2 may be useful for excluding some bipartite graphs from being standard, but we do not have any example to illustrate this.

We will, in fact, consider a slightly weaker condition of approximate finite dimensionality than the one in 3.1.

Definition 3.5. $N \subset M$ is AFD if $\forall x_{1}, \ldots, x_{n} \in M, \forall \varepsilon>0, \exists$ a finite dimensional inclusion $Q \subset P$ in $N \subset M$ such that $E_{N} E_{P}=E_{P} E_{N}=$ $E_{Q}, x_{i} \in \underset{\varepsilon}{\in} P, \forall i$.

We also consider the following functional analytical objects:

Definition 3.6. Let $\pi$ be a representation of $\mathfrak{U}=C_{\text {bin }}^{*}\left(M, e_{N}, M^{\text {op }}\right)$ on $\mathcal{B}(\mathcal{H})$. A state $\varphi$ on $\mathcal{B}(\mathcal{H})$ such that $\varphi(x T)=\varphi(T x), \forall x \in \pi(\mathfrak{U}), \forall T \in \mathcal{B}(\mathcal{H})$ 
is called a symmetric Markov hypertrace for $\mathfrak{U}$ via $\pi$. The term "hypertrace" is inspired from Connes' single algebra terminology. Yet note that if $N=M$ and $M, M^{o p}$ are represented on $\mathcal{B}\left(L^{2}(M)\right)$ in the usual way, then the above symmetric hypertraces $\varphi$ require both $M$ and $M^{o p}(=J M J)$ be in the centralizer of $\varphi$.

The next observation shows how one can "transport" hypertraces from a Hilbert space to another.

Proposition 3.7. If $\mathfrak{U}$ has a symmetric hypertrace via some representation $\pi_{0}$ then it has a symmetric hypertrace via any other representation $\pi$ of $\mathfrak{U}$ with $\operatorname{ker} \pi \subset \operatorname{ker} \pi_{0}$. In particular, if $C_{\min }^{*}\left(M, e_{N}, M^{\mathrm{op}}\right)$ has a symmetric hypertrace via some representation $\pi_{0}$ then any representation of $\mathfrak{U}$ has a symmetric hypertrace.

Indeed, if $\pi_{0}: \mathfrak{U} \rightarrow \mathcal{B}\left(\mathcal{H}_{0}\right)$, with $\varphi_{0}$ a state on $\mathcal{B}\left(\mathcal{H}_{0}\right)$ having $\pi_{0}(\mathfrak{U})$ in its centralizer, and $\pi: \mathfrak{U} \rightarrow \mathcal{B}(\mathcal{H})$ is another representation with $\operatorname{ker} \pi \subset \operatorname{ker} \pi_{0}$ then let $\sigma$ be the $*$-morphism from $\pi(\mathfrak{U})$ onto $\pi_{0}(\mathfrak{U})$ defined by $\sigma=\pi_{0} \circ$ $\pi^{-1}$ and $\psi$ a completely positive map from $\mathcal{B}(\mathcal{H})$ into $\mathcal{B}\left(\mathcal{H}_{0}\right)$ extending $\sigma$ (cf. Arveson's extension theorem). Then $\psi(T x)=\psi(T) \sigma(x), \psi(x T)=$ $\sigma(x) \psi(T)$ for any $x \in \pi(\mathfrak{U}), T \in \mathcal{B}(\mathcal{H})$. Thus $\varphi_{0} \circ \psi$ is a hypertrace for $\mathfrak{U}$ via $\pi$.

This result enables us to give one more characterization for the amenability of an inclusion.

Theorem 3.8. Let $N \subset M$ be an extremal inclusion. The following conditions are equivalent:

(i) $N \subset M$ is amenable.

(ii) If $\tau$ denotes the trace on $C_{\min }^{*}\left(M, e_{N}, M^{\mathrm{op}}\right)$ then $\pi_{\tau}$ has a symmetric Markov hypertrace.

(iii) Any representation $\pi$ of $C_{\mathrm{bin}}^{*}\left(M, e_{N}, M^{\mathrm{op}}\right)$ has a symmetric Markov hypertrace.

(iv) $C_{\mathrm{bin}}^{*}\left(M, e_{N}, M^{\mathrm{op}}\right)$ is simple.

Note that in the case $N=M$ the equivalence between (i) on (iv) reduces to some well known results of Effros-Lance. Also, related to (iv) in the above theorem, note that in ([Bi1]) the possibility for the compact operators $\mathcal{K}\left(L^{2}(M)\right)$ to be an ideal of $C^{*}\left(M, e_{N}, J M J\right)$ is proved equivalent to the existence of nontrivial central sequences for $M$ contained in $N$ (the " $N \subset$ $M$ " analogue of Connes' characterization of property $\Gamma$ ).

To prove 3.8 , note that by 2.1 , if $N \subset M$ is amenable then

$$
\overline{\pi_{\tau}\left(C_{\min }^{*}\left(M, e_{N}, M^{\mathrm{op}}\right)\right)}=M \underset{e_{N}}{\bigotimes} M^{\mathrm{op}}
$$


is hyperfinite so (i) $\Rightarrow$ (ii) and since the proof of (iv) $\Rightarrow(\mathrm{v})$ in 2.1 uses only the existence of a state having $\pi_{\tau}\left(C_{\min }^{*}\left(M, e_{N}, M^{\text {op }}\right)\right)$ in its centralizer, we also have (ii) $\Rightarrow$ (i). Then (ii) $\Leftrightarrow$ (iii) by 3.7. Next, if $x \in$ $C_{\mathrm{bin}}^{*}\left(M, e_{N}, M^{\mathrm{op}}\right) \subset \mathcal{B}(\mathcal{H})$ and $x$ is approximated in norm by an element of the form $x_{0}=\sum_{i=1}^{n} y_{i}^{\mathrm{op}} z_{i} x_{i}^{\mathrm{op}} \in s p M^{\mathrm{op}} M_{k} M^{\mathrm{op}}$ (cf. 1.2), then each $z_{i}$ can be approximated by $z_{i}^{\prime}=\sum_{j} p_{j} E_{\left(N_{\ell_{j}}^{j}\right)^{\prime} \cap M_{k}}^{M_{k}}\left(z_{i}\right) p_{j}$, for some choices of tunnels $\left\{N_{r}^{j}\right\}_{r}$ and $p_{j} \in \mathcal{P}\left(N_{\ell_{j}}^{j}\right) \mathcal{P}\left(N_{\ell_{j}}^{j}{ }^{\prime} \cap M_{k}\right)$, in the strong topology on $\mathcal{B}(\mathcal{H})$. Thus $x_{0}^{\prime}=\sum_{i} y_{i}^{\mathrm{op}} z_{i}^{\prime} x_{i}^{\mathrm{op}}$ approximate $x$ in the $*$-strong topology and $\left\|x_{0}^{\prime}\right\| \leq\|x\|$. But $\left\|x_{0}^{\prime}\right\|=\sup \left\|x_{0}^{\prime} p_{j}\right\|$ and each $\left\|x_{0}^{\prime} p_{j}\right\|$ is majorized by $\left\|\sum_{i} y_{i}^{\mathrm{op}} E_{N_{\ell_{j}}^{j} \cap M_{k}}^{M_{k}}\left(z_{i}\right) x_{i}^{\mathrm{op}}\right\|_{\mathcal{B}(\mathcal{H})}{ }^{j}=\left\|\sum_{i} y_{i}^{\mathrm{op}} E_{N_{\ell_{j}}^{j} \cap M_{k}}^{M_{k}}\left(z_{i}\right) x_{i}^{\mathrm{op}}\right\|_{M_{\ell_{j}}^{\mathrm{op}}}=$ $\left\|\sum_{i} y_{i}^{\mathrm{op}} E_{N_{\ell_{j}}^{j} \cap M_{k}}^{M_{k}}\left(z_{i}\right) x_{i}^{\mathrm{op}}\right\|_{C_{\min }^{*}\left(M, e_{N}, M^{\mathrm{op}}\right)} \leq\left\|x_{0}\right\|_{C_{\min }^{*}\left(M, e_{N}, M^{\mathrm{op}}\right)}$.

Thus, by the semicontinuity of the norm in the strong topology, we get $\|x\|_{\mathcal{B}(\mathcal{H})} \leq\|x\|_{C_{\min }^{*}\left(M, e_{N}, M^{\mathrm{op}}\right)}$. This proves (iii) $\Rightarrow$ (iv). Conversely, if (iv) holds true then a similar proof as in ([E-L]) shows that there exists a symmetric Markov hypertrace on $C^{*}\left(M, e_{N}, J M J\right) \subset \mathcal{B}\left(L^{2}(M)\right)$ and by 3.7 we get (iv) $\Rightarrow$ (ii).

We will now relate the existence of symmetric hypertraces to AFD properties of the subfactor.

Theorem 3.9. Let $N \subset M$ be an extremal inclusion of hyperfinite factors.

(a) If $\forall \varepsilon>0$ there exists a subfactor $Q \subset N$ such that $Q^{\prime} \cap N \subset Q^{\prime} \cap M$ is a finite dimensional inclusion with $\left\|T_{Q^{\prime} \cap N \subset Q^{\prime} \cap M}\right\|^{2}>[M: N]-\varepsilon$ then $N \subset M$ has the AFD property. If in addition $Q$ can be taken such that $[N: Q]<\infty$ then $N \subset M$ follows amenable. Also, if $[N: Q]<\infty$ and $\left\|T_{Q^{\prime} \cap N \subset Q^{\prime} \cap M}\right\|^{2}=[M: N]$ then $N \subset M$ has finite depth.

(b) If $N \subset M$ is $A F D$ then $C^{*}\left(M, e_{N}, J M J\right) \subset \mathcal{B}\left(L^{2}(M)\right)$ has a symmetric Markov hypertrace.

(c) $C^{*}\left(M, e_{N}, J M J\right)$ has a symmetric Markov hypertrace iff given any unitary elements $u_{1}, \ldots, u_{n} \in M$ and any $\varepsilon>0$ there exists a finite dimensional vector subspace $\mathcal{H}_{0} \subset L^{2}(M)$ such that $\mathcal{H}_{0}=$ $\mathcal{H}_{0}^{*}\left(=J \mathcal{H}_{0}\right), e_{N} \mathcal{H}_{0} \subset \mathcal{H}_{0}$ and

$$
\begin{aligned}
& \left\|u_{i} \operatorname{proj}_{\mathcal{H}_{0}} u_{i}^{*}-\operatorname{proj}_{\mathcal{H}_{0}}\right\|_{H S}<\varepsilon\left\|\operatorname{proj}_{\mathcal{H}_{0}}\right\|_{H S} \\
& \left\|J u_{i}^{*} \operatorname{pproj}_{\mathcal{H}_{0}} J u_{i} J-\operatorname{proj}_{\mathcal{H}_{0}}\right\|_{H S}<\varepsilon\left\|\operatorname{proj}_{\mathcal{H}_{0}}\right\|_{H S} .
\end{aligned}
$$


The proof of the first part of (a) is the same as the proof of the main theorem in [Po2], by using the hyperfiniteness of $N, M$ for approximate innerness and the hyperfiniteness of $Q$ for central freeness. The case when $[N: Q]<\infty$ is just a reformulation of 2.8 (ii). To prove (b), for any finite set $\alpha \subset M$ one considers an inclusion $Q_{\alpha} \subset P_{\alpha}$ such that $x \underset{1 /|\alpha|}{\varepsilon} P_{\alpha}, \forall x \in \alpha$, and such that each irreducible part of the inclusion matrix of $Q_{\alpha} \subset P_{\alpha}$ checks a Folner type condition as in [Po2] globally (it is easy to see that AFD implies one can do this). But then $\varphi(T)=\lim _{\alpha \rightarrow \omega}\left(\operatorname{dim} P_{\alpha}\right)^{-1} \operatorname{Tr}\left(\operatorname{Te} P_{P_{\alpha}}\right)$ will be a symmetric Markov hypertrace.

To prove (c) one uses the Day-Namioka-Connes trick like in (4.2 of [Po1]).

From (c) of the above theorem we can can now construct examples of hyperfinite subfactors without the AFD property, from certain classes of inclusions of the form $N^{\sigma} \subset M^{\sigma}$. Note that such an inclusion is AFD if and only if given any finite subst $X$ in $P$ and any $\varepsilon>0$, there exists a finite dimensional subalgebra $B$ in $P$ and unitary elements $u_{g_{1}}, \ldots, u_{g_{n}}$ in $B$ such that $X$ is $\varepsilon$-contained in $B$ and $\left\|A d u_{g_{i}}(x)-\sigma\left(g_{i}\right)(x)\right\|_{2}<\varepsilon$.

Corollary 3.10. Let $P$ be the hyperfinite type $\mathrm{II}_{1}$ factor, $G$ a property $T$ group and $\sigma$ a Bernoulli shift action on $P \simeq \underset{g \in G}{\otimes} R_{g}, R_{g} \simeq R, \forall g \in G$. Let $N^{\sigma} \subset M^{\sigma}$ be the locally trivial diagonal subfactor associated to $\sigma$ and to some choice of generators (5.1.5 in [Po1]). Then $C^{*}\left(M^{\sigma}, e_{N^{\sigma}}, M^{\sigma \mathrm{op}}\right) \subset$ $\mathcal{B}\left(L^{2}\left(M^{\sigma}\right)\right)$ has no symmetric Markov hypertraces. In particular, $N^{\sigma} \subset$ $M^{\sigma}$ is not $A F D$.

Indeed, by (c) of 3.9 we deduce that if a symmetric Markov hypertrace exists then the trivial representation of $G$ is weakly contained (thus, by property $\mathrm{T}$, actually contained) in the representation implemented by $\sigma$ on $L^{2}(P) \bar{\otimes} L^{2}\left(P^{\mathrm{op}}\right) \ominus \mathbb{C}$, contradiction.

Example 3.11. The (non)AFD property for subfactors of the form $N^{\sigma} \subset$ $M^{\sigma}$ is not always dictated by the nature of its standard graph $\Gamma_{N^{\sigma}, M^{\sigma}}$ $\left(=\right.$ Cayley matrix of $\left.\left(G ; g_{0}, g_{1}, \ldots, g_{n}\right)\right)$. To see this we consider a class of nonstandard AFD subfactors of the form $N^{\sigma} \subset M^{\sigma}$ as follows: Let $\left(G ; g_{0}, \ldots, g_{n}\right)$ be a finitely generated nonamenable group of finite resolution and $\pi_{n}: G \rightarrow G_{n}$ be a sequence of group morphisms from $G$ onto some finite groups $G_{n}$ such that $\cap \operatorname{ker} \pi_{n}=\{e\}$. Recall that free groups and most of the known property $\mathrm{T}$ groups have this property. Let $P=\otimes \mathcal{B}\left(l^{2}\left(G_{n}\right)\right)$ be the hyperfinite type $\mathrm{II}_{1}$ factor and let $\sigma$ be the properly outer action of $G$ on $P$ given by the product type action $\sigma(g)=\otimes \operatorname{Ad} \lambda_{n}\left(\pi_{n}(g)\right)$, where $\lambda_{n}$ is the left regular representation of $G_{n}$ on $l^{2}\left(G_{n}\right)$. Then $N^{\sigma} \subset M^{\sigma}$ is clearly AFD and if one takes $G=S L(n, Z), n>2$, then $G$ has the property $\mathrm{T}$ like the groups in 3.10, so that the standard graph of $N^{\sigma} \subset M^{\sigma}$ here will 
be the same as the one in 3.10. Yet one of this inclusions is AFD while the other is not.

Finally, let us mention that the reverse implications in (a) and (b) of 3.9 will be discussed in a forthcoming paper, dealing with the values of the index for the irreducible inclusions of hyperfinite type $\mathrm{II}_{1}$ subfactors.

\section{References}

[Bi1] D. Bisch, On the existence of central sequences in subfactors, Trans. Amer. Math. Soc. 321 (1990), 117-128.

[Bi2] _ A note on intermediate subfactors, Pac. J. Math. (to appear).

[C1] A. Connes, Classification of injective factors, Ann. Math. 104 (1976), 73-115.

[C2] Compact metric spaces, Fredholm modules and hyperfiniteness, Pacific J. Math. 137 (1989), 120-135.

[EL] E. Effros and C. Lance, Tensor products of operator algebras, Advances in Math. 25 (1977), 1-34.

[dlHJ] P. de la Harpe and V. F. R. Jones, Paires de sous-algebres semi-simples et graphes fortement reguliers, C. R. Acad. Sci. Paris (1990).

[H] U. Haagerup, in preparation.

[HSc] U. Haagerup and J. Schou, Some new subfactors of the hyperfinite type $\mathrm{II}_{1}$ factor, preprint (1991).

[Hi] F. Hiai, Entropy and growth for derived towers of subfactors, preprint (1993).

[J] V. F. R. Jones, Index for subfactors, Invent. Math. 72 (1983), 1-25.

[PiPo1] M. Pimsner and S. Popa, Entropy and index for subfactors, Ann. Sci. Ec. Norm. Sup. 19 (1986), 57-106.

[PiPo2] _ Finite dimensional approximation for pairs of algebras and obstructions for the index, J. Funct. Analysis 98 (1991), 270-291.

[Po1] S. Popa, Classification of amenable subfactors of type II, Acta. Math. 172 (1994), 253-345.

[Po2] , Approximate innerness and central freeness for subfactors: a classification result, preprint (1993).

[Po3] - Subfactors and classification results in von Neumann algebras in Proc. ICM Kyoto 1990, Springer Verlag, Tokyo, 1991, pp. 987-996.

[We] H. Wenzl, Hecke algebras of type $A_{n}$ and subfactors, Invent. Math. 92 (1988), 349-383.

Dept. of Math., Univ. of California, Los Angeles, CA 90024-1555

E-mail address: popa@math.ucla.edu 